\title{
Analysis of the Brinkman equation as a model for flow in porous media
}

\author{
L. Durlofsky and J. F. Brady \\ Department of Chemical Engineering, California Institute of Technology, Pasadena, California 91125
}

(Received 21 May 1987; accepted 17 July 1987)

\begin{abstract}
The fundamental solution or Green's function for flow in porous media is determined using Stokesian dynamics, a molecular-dynamics-like simulation method capable of describing the motions and forces of hydrodynamically interacting particles in Stokes flow. By evaluating the velocity disturbance caused by a source particle on field particles located throughout a monodisperse porous medium at a given value of volume fraction of solids $\phi$, and by considering many such realizations of the (random) porous medium, the fundamental solution is determined. Comparison of this fundamental solution with the Green's function of the Brinkman equation shows that the Brinkman equation accurately describes the flow in porous media for volume fractions below 0.05 . For larger volume fractions significant differences between the two exist, indicating that the Brinkman equation has lost detailed predictive value, although it still describes qualitatively the behavior in moderately concentrated porous media. At low $\phi$ where the Brinkman equation is known to be valid, the agreement between the simulation results and the Brinkman equation demonstrates that the Stokesian dynamics method correctly captures the screening characteristic of porous media. The simulation results for $\phi \geqslant 0.05$ may be useful as a basis of comparison for future theoretical work.
\end{abstract}

\section{INTRODUCTION}

Averaged equations describing viscous flow through porous media are of great theoretical and practical interest. At the fundamental microscale the Stokes equations apply and provide a complete description of the entire flow field. However, as a result of the complex and often only statistically known geometry of the solid surfaces in the medium, solution of the Stokes equations is generally very difficult. On the macroscopic level, Darcy's law, first established empirically but more recently derived formally by performing appropriate volume averages of the Stokes equations, is applicable. ${ }^{1-4}$ The qualitative difference between these two descriptions of the flow motivated Brinkman ${ }^{5}$ to suggest a general equation that interpolates between the Stokes equation and Darcy's law. His equation,

$$
\mu \nabla^{2} \mathbf{u}-\nabla p-\mu \alpha^{2} \mathbf{u}=0, \quad \nabla \cdot u=0,
$$

where $\mu$ is the Newtonian fluid viscosity, $\alpha^{-2}$ is the permeability, and $u$ and $p$ are the average velocity and pressure, is, like the Stokes equation but unlike Darcy's law, second order in velocity. This is significant since it allows for the solution of flow around a particle or flow caused by motion of a particle with no-slip boundary conditions on the surface. The averages implicit in (1) should be viewed as averages over an ensemble of different realizations of the porous medium.

On small length scales in the Brinkman equation, the pressure gradient balances the Laplacian of the velocity and the flow is essentially viscous. Over larger length scales, where the velocity is slowly varying, the pressure gradient balances the average velocity as it does in Darcy's law. The characteristic length that distinguishes between these two regions of scaling is the Brinkman screening length given by the square root of the permeability $\alpha^{-1}$. In the dilute limit $\alpha^{-1}=(\sqrt{2} / 3) a \phi^{-1 / 2}$, where $a$ is the characteristic particle size and $\phi$ is the volume fraction of solids. In a viscous fluid, the velocity disturbance resulting from a point force decays as $1 / r$, where $r$ is the distance from the source point to a point in the fluid. At large distances $\left(r>\alpha^{-1}\right)$ from a point force disturbance in a Brinkman medium, however, the response is very different, with the velocity disturbance decaying faster, as $1 /\left(\alpha^{2} r^{3}\right)$.

Though Brinkman's derivation of Eq. (1) was heuristic, subsequent investigators have rigorously established the validity of this equation at low volume fraction of solids. ${ }^{6-12}$ The question of the applicability of the Brinkman equation, i.e., how well it describes flow behavior in a porous medium, at higher values of $\phi$ remains open, however, although theoretical predictions of permeability based on the Brinkman equation agree well with experimentally measured values. ${ }^{5,13}$ This in itself does not establish its validity (even empirically) for other than dilute systems, as the permeability is only a single scalar quantity and is not necessarily representative of the general flow field.

In this paper we employ Stokesian dynamics to approximate the fundamental solution, or Green's function, for flow in random porous media. Stokesian dynamics is a general, molecular-dynamics-like simulation method capable of describing the motions of, or forces resulting from, hydrodynamically interacting particles immersed in a viscous fluid under conditions of vanishing particle Reynolds number. The general method applied to finite systems of hydrodynamically interacting particles has been presented recently by Durlofsky, Brady, and Bossis ${ }^{14}$; extensions to infinite systems, required for the present problem, are considered by Brady $e t$ $a l .{ }^{15}$ Once the fundamental solution is determined via Stokesian dynamics it can be compared with the solution of Brinkman's equation, allowing an assessment of the applicability of the Brinkman equation to porous media of arbitrary volume fraction. 
We discuss the application of the general Stokesian dynamics methodology to the present problem in Sec. II. There it will be seen that the problem is essentially to form the $N$ particle mobility matrix, which relates the difference between the velocity of each particle and the suspension average velocity to the forces exerted by each particle on the fluid. Due to the slowly decaying nature of particle interactions in Stokes flow, the effects of particles a great distance from a given particle must be included. This is accomplished efficiently using the Ewald summation technique, recently presented for Stokes flow by Beenakker, ${ }^{16}$ and shown to yield a convergent result by applying the method developed by $\mathrm{O}^{\prime} \mathrm{Brien},{ }^{17}$ as in Brady et al. ${ }^{15}$ Appropriate manipulations and averages of this mobility matrix result in the fundamental solution as well as the permeability for the system in question.

In Sec. III we compare our simulation results for the fundamental solution with the Brinkman propagator or Green's function. Our results are for systems of both point forces and identical finite-sized spheres, computed both with and without the application of the Ewald summation technique. For very dilute systems $(\phi \leqslant 0.01)$, the simulation results for the fundamental solution computed with Ewald sums agree very well with the Green's function for the Brinkman equation. Because the Brinkman equation is valid as $\phi \rightarrow 0$ this is to be expected and serves as a verification of our method. Discrepancies between the Brinkman and simulation results are evident in simulations performed without Ewald sums, illustrating the importance, even at low $\phi$, of effects from distant particles. In moderately dilute systems, $\phi=0.05$, the fundamental solution is still well described by the Brinkman propagator, though differences are clearly apparent. The Brinkman result is seen to be no longer quantitatively applicable in moderately concentrated systems $\phi$ $=0.2$, though it still provides a qualitative picture of particle interactions in a porous medium. Finally, we present results for permeability that agree well with the results of Brinkman $^{5}$ and Kim and Russel. ${ }^{13}$

\section{DETERMINATION OF THE FUNDAMENTAL SOLUTION FOR FLOW IN POROUS MEDIA}

Our intent is to formulate a method that will allow us to determine the velocity field due to a point force disturbance in an unbounded porous medium. The disturbance (source) is applied at a given position and the suspension velocity (response) is measured at all points (field points) within the porous medium. By repeating this procedure for many different realizations of the porous medium and performing appropriate averages, the form of the fundamental solution for flow in porous media can be deduced. The particles that comprise the porous medium are prescribed to remain fixed in space though the forces that they must exert on the fluid to remain so are unknown. For a given velocity disturbance at some point in the fluid, the force that any particular particle must exert to remain fixed depends in part on the positions of the other particles in the system. Thus the system is fully coupled; the response of each individual particle is affected by every other particle.

In all that follows, we suppose that the flow on the parti- cle scale is in the Stokes regime; i.e., that the particle Reynolds number $U a / v$, where $U$ is a characteristic velocity, $a$ the characteristic particle size, and $v$ the kinematic viscosity of the fluid, is much less than unity.

\section{A. Formation of the $\boldsymbol{N}$-particle mobility matrix}

In Stokes flow problems involving systems of hydrodynamically interacting particles, the velocities of all the particles can be related to the forces exerted on the fluid by each of the particles via the mobility matrix $\mathbf{M}$ :

$$
\mathbf{U}=\mathbf{M} \cdot \mathbf{F} \text {, }
$$

where $\mathbf{U}$ is the translational/rotational velocity vector and $\mathbf{F}$ is the force/torque vector, for all $N$ particles in the system. The inverse problem involves the resistance matrix $\mathbf{R}$, which relates force/torques to particle velocities;

$$
\mathbf{F}=\mathbf{R} \cdot \mathbf{U} \text {. }
$$

The resistance matrix is the inverse of the mobility matrix,

$$
\mathbf{R}=\mathbf{M}^{-1} \text {. }
$$

Here, $M$ and $\mathbf{R}$ depend only on the instantaneous particle configuration; they are not affected by the velocities or forces imposed on the particles. In addition, both $\mathbf{M}$ and $\mathbf{R}$ are symmetric and positive definite.

General methods have recently been presented for accurately approximating $\mathbf{R}$ (and therefore $\mathbf{M}$ ) for both finite and infinite systems of hydrodynamically interacting particles. ${ }^{14,15}$ Because these methods are applied to dynamically evolving configurations of particles, substantial effort is required to assure that short-range lubrication forces, which act to prevent particles from overlapping during the course of a dynamic simulation, are correctly included in the resistance matrix. In the present problem, however, only static (i.e., instantaneous) configurations need be considered, and our interest is in long-range effects, which are not influenced by lubrication forces. Thus considerable simplification from the more general approaches is possible in the present case.

We begin the development of the method by considering the formulation for a finite system of particles and then pass to the thermodynamic limit; i.e., let the number of particles $N$ and the volume of the system $V$ approach infinity keeping the ratio $N / V$ constant. The subsequent development is limited to systems of either point forces or identical spheres of radius $a$, although it is straightforward to generalize to more complex systems. Performing a moment expansion of the integral representation for the Stokes flow velocity field and applying Faxén's law, we have the following relationship between the translational velocity of a given sphere, with center at $\mathbf{x}_{\alpha}$, and the other $N-1$ spheres ${ }^{14}$ :

$$
\begin{aligned}
\mathbf{U}^{\alpha}-\mathbf{u}^{\infty}\left(\mathbf{x}_{\alpha}\right)= & \frac{\mathbf{F}^{\alpha}}{6 \pi \mu a}+\frac{1}{8 \pi \mu} \\
& \times \sum_{\substack{\beta=1 \\
\beta \neq \alpha}}\left(1+\frac{a^{2}}{3} \nabla^{2}\right) \mathbf{J}\left(\mathbf{x}_{\alpha}-\mathbf{x}_{\beta}\right) \cdot \mathbf{F}^{\beta},
\end{aligned}
$$

where $\mathbf{U}^{\alpha}$ is the velocity of sphere $\alpha, \mathbf{u}^{\infty}\left(\mathbf{x}_{\alpha}\right)$ is the imposed flow at infinity evaluated at the sphere center, $\mathbf{F}^{\alpha}$ is the force 
exerted by sphere $\alpha$ on the fluid, and $\mathbf{J}$ is the free-space Green's function or fundamental solution for Stokes flow,

$$
\mathrm{J}(\mathbf{r})=\mathbf{I} / r+\mathbf{r r} / r^{3},
$$

where $\mathbf{r}=\mathbf{x}_{\alpha}-\mathbf{x}_{\beta}$ and $r=|\mathbf{r}|$. For point forces, the $\left(a^{2} /\right.$ 3) $\nabla^{2} \mathrm{~J}$ term is identically zero. Writing (5) for each of the $N$ particles in the system, an approximation to the mobility matrix of Eq. (2) can be constructed. For systems of finitesized spheres, each sphere-sphere interaction is simply the well-known Rotne-Prager tensor, the long-range part of the complete two-sphere interaction, evaluated as though the two spheres were alone in the fluid. Effects from third bodies do not affect the two-sphere interactions until $O\left(1 / r^{4}\right)$, where $r$ is a characteristic particle spacing. This is consistent with the Rotne-Prager approximation, which also neglects terms of $O\left(1 / r^{4}\right)$ in the two-sphere interactions. For systems of point forces, Eq. (5), without the finite size $\left(a^{2} / 3\right) \nabla^{2} \mathbf{J}$ term, is exact; no higher-body effects at all enter in the mobility matrix.

Inversion of the $N$-particle mobility matrix formed as described above gives a far-field approximation to the resistance matrix of (3). As discussed by Durlofsky et al., ${ }^{14}$ inverting the mobility matrix actually performs all the manybody reflections among all particles. Thus, although the mobility matrix is formed in a pairwise-additive manner, its invert, the resistance matrix, contains many-body interactions. In fact, it is these many-body reflections, summed upon the inversion of the mobility matrix, that give rise to the screening characteristic of porous media. In the resistance matrix, two-body interactions are via a medium of fixed (nonzero force) particles, in contrast to the mobility matrix, where two-body interactions are via a medium of force-free (nonzero velocity) particles. Therefore, the resistance interactions provide precisely the type of information required to extract the form of the fundamental solution in porous media.

The discussion up to this point has been limited to systems of finite numbers of particles. We now consider the extensions required for infinite systems. To pass to the thermodynamic limit, the number of particles $N$ and the volume of the system $V$ approach infinity with the ratio $N / V$ constant. Thus, the volume fraction of particles

$$
\phi=\frac{4}{3} \pi a^{3} N / V,
$$

for spherical particles of radius $a$, can be defined. To simulate an infinite system we could, theoretically, form the mobility matrix as described above for a system of $N$ spheres and focus only on a subsystem of $N_{1}$ spheres immersed within the larger system. For sufficiently large $N\left(N \gg N_{1} \gg 1\right)$, such a system should be representative of an unbounded suspension; in fact, a rigorously convergent expression can be constructed as $N$ and $N_{1} \rightarrow \infty$. This type of approach is, however, very inefficient computationally; $N$ particles must be included in the simulation but only $N_{1}$ particles provide any information. Instead of proceeding as described above, we impose periodic boundary conditions, a technique widely used in molecular dynamics and Monte Carlo simulations. This means that we focus on a system of $N$ particles contained within a cell that is periodically replicated throughout all space. Taking the periodic cell to be a cube of side $H$, the volume fraction of particles $\phi$ is given by Eq. (7) with $H^{3}$ replacing $V$. Although periodic boundary conditions are imposed out of computational necessity, our intent is to model random systems. Thus the periodic cell must be large enough that positions of neighboring particles appear random to a particle placed at the center of the periodic cell. This point will be discussed in more detail below. In the simulation results discussed in Sec. III we shall refer to systems of point forces at nonzero volume fractions. What this means is simply that the particles interact as point forces; i.e., without the finite-size term in Eq. (5). They are still physically spheres of radius $a$, and occupy a nonzero volume, with a well-defined volume fraction as given by Eq. (7).

Because the sphere mobility interactions decay slowly, as $1 / r$, the effects of spheres far from the test sphere must be included in the simulation. Therefore, spheres in a given periodic cell must interact not only with spheres in the same periodic cell but also with the images in other periodic cells. This entails performing so-called lattice sums of the RotnePrager tensor of Eq. (5). Thus for a system of $N$ particles replicated periodically in space, Eq. (5) becomes, with no impressed flow at infinity,

$$
\begin{aligned}
\mathbf{U}^{\alpha}= & \frac{\mathbf{F}^{\alpha}}{6 \pi \mu a}+\frac{1}{8 \pi \mu} \\
& \times \sum_{\gamma} \sum_{\beta=1}\left(1+\frac{a^{2}}{3} \nabla^{2}\right) \mathbf{J}\left(\mathbf{x}_{\alpha}-\mathbf{x}_{\beta}\right) \cdot \mathbf{F}^{\beta},
\end{aligned}
$$

where $\gamma$ numbers the unit cells and the double summation is not performed for $\gamma=1, \beta=\alpha$.

Beenakker ${ }^{16}$ applied the Ewald summation technique, previously used to handle slowly decaying Coulombic interactions, to these Rotne-Prager lattice sums, casting them into a rapidly converging form. In his development, Beenakker specified that the total force on the particles in a unit cell be zero, and thus the infinite sum in ( 8$)$ is well defined and convergent. It is essential that the convergence of these sums be accelerated, as the slow decay of the interaction would result in very slowly converging sums at great computational cost and questionable accuracy. Writing the Ewaldsummed version of Eq. (8) for each of the $N$ particles in the periodic cell (see Beenakker ${ }^{16}$ for details), an approximation to the mobility matrix can now be constructed that is valid for $N$ particles immersed in an unbounded system. We designate the mobility matrix to which this Ewald summation technique is applied $\mathbf{M}^{*}$ and write

$$
\mathbf{U}=\mathbf{M}^{*} \cdot \mathbf{F} \text {. }
$$

Note that the mobility matrix in Eq. (9), as well as all subsequent mobility and resistance matrices, relate translational velocity and force, in contrast to the more general matrices in Eqs. (2)-(4), which relate translational/rotational velocities to force/torque. Similarly, $U$ now designates the particle translational velocity vector and $F$ the force vector.

When the average force the particles exert on the fluid is not zero, the expression ( 8 ) for the velocity of a particle must be modified. This can be accomplished in a rigorous fashion by applying a technique first proposed by O'Brien ${ }^{17}$ to an infinite suspension of forced particles. The details of such an approach are in Brady et al. ${ }^{15}$; here we shall only 
sketch the derivation. We start from an integral representation for the solution to Stokes equations for the velocity field $\mathbf{u}(\mathbf{x})$ at a point $\mathbf{x}$ in the fluid in terms of integrals of the force distribution on the particle surfaces and an integral over a mathematical surface $\Gamma$ of large radius that cuts through both fluid and particles:

$$
\begin{aligned}
\mathbf{u}(\mathbf{x})= & -\frac{1}{8 \pi \mu} \sum_{\alpha=1}^{N} \int_{S_{\alpha}} \mathbf{J} \cdot \boldsymbol{\sigma} \cdot \mathbf{n} d S \\
& -\frac{1}{8 \pi \mu} \int_{S_{\mathbf{r}}}(\mathbf{J} \cdot \boldsymbol{\sigma}+\mathbf{K} \cdot \mathbf{u}) \cdot \mathbf{n} d S .
\end{aligned}
$$

Here $\mathbf{J}$ is the Green's function (6), $\mathbf{K}=-6 \mu \mathrm{rrr} / r^{5}$, $\mathbf{r}=\mathbf{x}-\mathbf{y}, \mathbf{y}$ being a point on the surface, $\sigma$ is the fluid stress tensor, and $\mathbf{n}$ is the outer normal to the surfaces.

Equation (10) is exact for rigid particles. No divergences occur because we have a finite region bounded by the surface $\Gamma$. This surface is an arbitrary surface immersed in an unbounded statistically homogeneous suspension, i.e., the suspension continues outside of $\Gamma$. If the radius of this surface is taken to be very large, the variation in $J$ and $K$ will be small over a surface element $d S_{\Gamma}$ that cuts many particles and fluid. Thus in the integrand of the second integral we may replace $\sigma$ and $\mathbf{u}$ by their suspension averages-fluid and particle phase averages- $\langle\sigma\rangle$ and $\langle u\rangle$. In a statistically homogeneous suspension, $\langle\sigma\rangle$ and $\langle u\rangle$ are either constants or linear functions of position, arising from the average pressure in $\langle\sigma\rangle$ and a linear shear flow (if one exists) in $\langle u\rangle$.

Using the divergence theorem, and introducing the suspension average quantities, (10) can be manipulated to give

$$
\begin{aligned}
\mathbf{u}(\mathbf{x})-\langle\mathbf{u}(\mathbf{x})\rangle= & -\frac{1}{8 \pi \mu} \sum_{\alpha=1}^{N} \int_{S_{\alpha}} \mathbf{J} \cdot \boldsymbol{\sigma} \cdot \mathbf{n} d S \\
& -\frac{n}{8 \pi \mu} \int_{0}^{R} \mathbf{J} \cdot\langle\mathbf{F}\rangle d V
\end{aligned}
$$

Here, $\langle\mathbf{F}\rangle$ is the average force the particles exert on the fluid, $n=N / V$ is the number density of particles, and $R$ is the radius of the volume enclosed by the surface $\Gamma$. The above reduction is valid for point forces only, but it is straightforward to generalize to the complete problem. ${ }^{15}$ It is now permissible to let $R \rightarrow \infty$, because at large distances from $\mathrm{x}$ the sum becomes equivalent to a volume integral of $n$ times the average force, and (11) results in a finite convergent expression for $\mathbf{u}-\langle\mathbf{u}\rangle$. Physically, the integral represents a "back flow" of fluid, relative to zero volume flux axes $\langle\mathbf{u}\rangle=0$, caused by the macroscopic pressure gradient that balances the excess weight, $\langle\mathbf{F}\rangle \neq 0$, of the particles. It is the velocity relative to this average back flow that is the physically significant quantity, not its absolute value.

Using the above procedure and Faxén laws for particle velocities, absolutely convergent expressions for the particle velocities in Eq. (8) can be obtained. With the obvious changes in notation on the particle sums and expanding the surface integrals in (10) moments, we have in lieu of (8)

$$
\begin{aligned}
\mathbf{U}^{\alpha}-\left\langle\mathbf{u}\left(\mathbf{x}_{\alpha}\right)\right\rangle= & \frac{\mathbf{F}^{\alpha}}{6 \pi \mu a}+\frac{1}{8 \pi \mu} \sum_{\gamma} \sum_{\beta=1}^{N}\left(1+\frac{a^{2}}{3} \nabla^{2}\right) \\
& \times \mathbf{J}\left(\mathbf{x}_{\alpha}-\mathbf{x}_{\beta}\right) \cdot \mathbf{F}^{\beta}-\phi \frac{\langle\mathbf{F}\rangle}{6 \pi \mu a} \\
& -\frac{n}{8 \pi \mu} \int_{0}^{\infty}\left(1+\frac{a^{2}}{3} \nabla^{2}\right) \mathbf{J} \cdot\langle\mathbf{F}\rangle d V .
\end{aligned}
$$

The constant term $\phi\langle\mathbf{F}\rangle$ results from the finite particle size contributions $\left(a^{2} / 3\right) \nabla^{2} \mathbf{J}$. [The reduction of (10) for finitesized particles requires some care and is discussed in Brady et al. ${ }^{15}$ ] Equation (12) is an absolutely convergent expression for the velocities of the $N$ particles that have been periodically replicated throughout all space.

It is not necessary for the particles to be periodically replicated. Equation (11) applies for any distribution of particles, but convergence of the difference between the sum and the integral in (11) or (12) can be accelerated by using periodic replication and the Ewald summation technique. Applying the Ewald summation procedure to (12) results in exactly the same Ewald-summed mobility matrix $\mathbf{M}^{*}$ as in Eq. (9). The only change the average force makes is to add the suspension average velocity to the left-hand side. Thus, in place of (9) when the particles are not force-free, we have

$$
\mathbf{U}-\langle\mathbf{u}\rangle=\mathbf{M}^{*} \cdot \mathbf{F} \text {. }
$$

Mathematically, the stipulation by Beenakker that $\langle\mathbf{F}\rangle=0$ removes a singular term in the reciprocal space lattice sum at $\mathbf{k}=0$, where $\mathbf{k}$ is a reciprocal wave vector. The constant term $\phi\langle\mathbf{F}\rangle$ and the back-flow integral in (12) precisely cancel this $\mathbf{k}=0$ term when the average force is not zero. It may appear surprising that the same mobility matrix $\mathbf{M}^{*}$ is obtained whether or not the average force is zero, but there is a simple intuitive argument that shows it must be true. The mobility matrix is a purely geometric quantity that describes particle interactions. It cannot depend on the velocity, forces, etc., that the particles ultimately have. Therefore, it must be the same whether or not the average force is zero. Said differently, in writing the mobility matrix in (13) the particles do not know whether the forces sum to zero or to a finite value; the particle interactions must be the same in the two cases.

The Ewald summed mobility matrix $\mathbf{M}^{*}$ contains all the information required to compute the fundamental solution in porous media. Before describing the manipulations that must be performed to extract the desired results, we shall briefly consider how $\mathbf{M}^{*}$ differs from the far-field mobility approximations of Durlofsky et al. and Brady et al. In the present problem, the mobility matrix relates translational velocities to forces; no angular velocities, imposed rate of strain, torques, or stresslets are included. In the formulations of Durlofsky et al. and Brady et al., these additional interactions are included. In the problem at hand, because no nonuniform bulk flow or external torques are imposed and because the particles are distributed randomly, the angular velocity, rate of strain, torque, and stresslet unknowns need not be included. This results in a significant computational savings; $\mathbf{M}^{*}$ is reduced from $11 N \times 11 N$ to $3 N \times 3 N$. 
We mention in passing that the procedure described above is fully applicable to ordered as well as random systems. The bulk properties of regular systems are studied in detail by Brady et al. ${ }^{15}$ through an approach much like that described here.

\section{B. Fundamental solution for flow in porous media and comparison with the Brinkman equation}

To determine the Green's function for flow in porous media, we should apply an infinitesimal point force at a point in the medium and measure the velocity response at all field points (both fluid and solid phase points since Brinkman's equation applies to suspension averages). This could be accomplished by using two "test" particles of very small radii in the mobility formulation (13), and then forming the mobility invert to approximate the $N$-particle resistance matrix $\mathbf{R}^{*}$ :

$$
\mathbf{F}=\mathbf{R}^{*} \cdot(\mathbf{U}-\langle\mathbf{u}\rangle) .
$$

Recall that interactions in the resistance matrix are via a bed of fixed particles. The Green's function can then be extracted from (14) as described below.

While correct, the above approach is computationally expensive because the procedure would have to be repeated for many different bed particle configurations and for many different test particle locations in order to form averages. Instead, we shall simply use two of the bed particles as the source and field points, applying a force to one and measuring the velocity of the other. For each configuration of bed particles all possible pairs of particles can be used as source and field points, and a large amount of statistical information can be obtained from a single realization. Thus, for two particles $\alpha$ and $\beta$, we form the two-particle resistance matrix corresponding to their interactions:

$$
\left[\begin{array}{l}
\mathbf{F}^{\alpha} \\
\mathbf{F}^{\beta}
\end{array}\right]=\left[\begin{array}{ll}
\mathbf{R}_{\alpha \alpha}^{*} & \mathbf{R}_{\alpha \beta}^{*} \\
\mathbf{R}_{\beta \alpha}^{*} & \mathbf{R}_{\beta \beta}^{*}
\end{array}\right] \cdot\left[\begin{array}{l}
\mathbf{U}^{\alpha}-\langle\mathbf{u}\rangle \\
\mathbf{U}^{\beta}-\langle\mathbf{u}\rangle
\end{array}\right]
$$

where $\mathbf{R}_{\alpha \alpha}^{*}$ and $\mathbf{R}_{\beta \beta}^{*}$ are the $3 \times 3$ self-term component matrices and $\mathbf{R}_{\alpha \beta}^{*}$ and $\mathbf{R}_{\beta \alpha}^{*}$ are $\alpha-\beta$ interaction matrices of the $N$ particle resistance matrix.

If a force is applied to one particle in a porous medium of infinite extent, the average suspension velocity $\langle\mathbf{u}\rangle$ as well as the total force exerted by the particles on the fluid, $\Sigma \mathbf{F}^{\alpha}$ $=N\langle\mathbf{F}\rangle$, are identically zero. Owing to the imposition of periodic boundary conditions in our model systems, however, the velocity disturbance is periodically replicated throughout all space, yielding, in general, nonzero values for the total force and the suspension average velocity. In simulation $\langle\mathbf{u}\rangle$ or $\langle\mathbf{F}\rangle$ must be specified to obtain a well-defined problem, and thus we set either $\langle\mathbf{u}\rangle \equiv 0$ or $\langle\mathbf{F}\rangle \equiv 0$. Note, it is not possible to prescribe both $\langle\mathbf{u}\rangle$ and $\langle\mathbf{F}\rangle$ because this overdetermines the system of equations. The simplification to (12) is obvious in the case $\langle\mathbf{u}\rangle \equiv 0$; for $\langle\mathbf{F}\rangle \equiv 0$ the following condition on $\langle\mathbf{u}\rangle$ can be derived from Eq. (14):

$$
\langle\mathbf{u}\rangle=\left(\sum_{\alpha=1}^{N} \sum_{\beta=1}^{N} \mathbf{R}_{\alpha \beta}^{*}\right)^{-1} \cdot\left(\sum_{\gamma=1}^{N} \mathbf{R}_{\gamma \delta}^{*}\right) \cdot \mathbf{U}^{\delta}
$$

for translation of particle $\delta$ with all other particles fixed. Averaging (16) over all particles $\delta,\langle u\rangle$ can be simply expressed as

$$
\langle\mathbf{u}\rangle=(1 / N) \mathbf{U},
$$

where $U$ is the translational velocity of any particle with all others fixed. Thus, for the case $\langle\mathbf{F}\rangle \equiv 0$, Eq. (15) becomes

$$
\left[\begin{array}{l}
\mathbf{F}^{\alpha} \\
\mathbf{F}^{\beta}
\end{array}\right]=\left[\begin{array}{ll}
\mathbf{R}_{\alpha \alpha}^{*}-(1 / N)\left(\mathbf{R}_{\alpha \alpha}^{*}+\mathbf{R}_{\alpha \beta}^{*}\right) & \mathbf{R}_{\alpha \beta}^{*}-(1 / N)\left(\mathbf{R}_{\alpha \alpha}^{*}+\mathbf{R}_{\alpha \beta}^{*}\right) \\
\mathbf{R}_{\beta \alpha}^{*}-(1 / N)\left(\mathbf{R}_{\beta \alpha}^{*}+\mathbf{R}_{\beta \beta}^{*}\right) & \mathbf{R}_{\beta \beta}^{*}-(1 / N)\left(\mathbf{R}_{\beta \alpha}^{*}+\mathbf{R}_{\beta \beta}^{*}\right)
\end{array}\right] \cdot\left[\begin{array}{l}
\mathbf{U}^{\alpha} \\
\mathbf{U}^{\beta}
\end{array}\right]
$$

Equation (18) above, valid for systems in which $\langle F\rangle$ $\equiv 0$, differs from the result for systems with $\langle\mathbf{u}\rangle \equiv 0$ by terms proportional to $1 / N$. Therefore, the results from two specifications will differ by an amount that tends to zero as $N \rightarrow \infty$, as would be expected physically. The convergence of the results from the two specifications with increasing $N$ will be clearly illustrated in Sec. III. Note that the expressions given above allow for nonzero $\langle\mathbf{u}\rangle$ even when $\langle\mathbf{F}\rangle \equiv 0$ and nonzero $\langle F\rangle$ when $\langle u\rangle \equiv 0$. These results, though nonintuitive, are consistent with a macroscopic momentum balance. Specifically, in the case $\langle\mathbf{u}\rangle \equiv 0,\langle\mathbf{F}\rangle \neq 0$, a macroscopic pressure gradient appears which generates an average fluid velocity that exactly balances the velocity generated by the periodically replicated translating particle. For the case $\langle u\rangle \neq 0$, $\langle F\rangle \equiv 0$, no macroscopic pressure gradient exists, even though a bulk flow is present, because none is required to balance the forces exerted by the particles on the fluid.

For a direct comparison of the two-particle simulation result with the Brinkman propagator, the two-particle resistance expressions, Eq. (15) for the case $\langle\mathbf{u}\rangle \equiv 0$ and Eq. (18) for the case $\langle F\rangle \equiv 0$, must be recast into mobility expressions, accomplished through inversions. The resulting mobility matrix for either case is designated as $\mathbf{M}_{\alpha \beta}^{P}$, where the superscript $P$ indicates that the two-particle interaction is via a porous medium.

The nondimensional velocity field created by a point force located at $\mathbf{x}_{\alpha}$ in a Brinkman medium is

$$
\mathbf{u}(\mathbf{x})=\frac{3}{4} \mathscr{J}\left(\mathbf{x}-\mathbf{x}_{\alpha}\right) \cdot \mathbf{F}^{\alpha},
$$

where $\mathbf{u}(\mathbf{x})$ is the velocity at a point $\mathbf{x}$ and $\mathbf{F}^{\alpha}$ is the force nondimensionalized by $6 \pi \mu a U$. The Brinkman propagator $\mathscr{J}$ is given by Howells ${ }^{8}$ as

$$
\begin{aligned}
\mathscr{J}= & \left(2 / \alpha^{2} r^{3}\right)\left[\left(1+\alpha r+\alpha^{2} r^{2}\right) e^{-\alpha r}-1\right] \mathbf{I} \\
& +\left(6 / \alpha^{2} r^{5}\right)\left[1-\left(1+\alpha r+\frac{1}{3} \alpha^{2} r^{2}\right) e^{-\alpha r}\right] \mathbf{r r},
\end{aligned}
$$


expressed more concisely as

$$
\mathscr{J}=\frac{4}{3} f_{\mathrm{B}}(r) \mathbf{I}+\frac{4}{3} g_{\mathrm{B}}(r)\left(\mathrm{rr} / r^{2}\right),
$$

where $f_{\mathrm{B}}(r)$ and $g_{\mathrm{B}}(r)$ are apparent from (20a), with the factor of $\frac{4}{3}$ introduced to cancel the $\frac{3}{4}$ in Eq. (19). In the limit $\phi \rightarrow 0, \alpha$, nondimensionalized by the sphere radius $a$, is given by

$$
\alpha=(3 / \sqrt{2}) \phi^{1 / 2} \text {. }
$$

Note that the velocity disturbance due to a point force in a Brinkman medium decays far away as $1 /\left(\alpha^{2} r^{3}\right)$, in contrast to the $1 / r$ decay of the Stokes propagator, which can be recovered from (20) in the limit $\alpha \rightarrow 0$. Also of interest is the observation that integration of $\mathscr{J}$ over a spherical surface results in an expression that decays exponentially with $r$ rather than algebraically.

For any isotropic, homogeneous medium, the propagator or fundamental solution can be expressed in a form analogous to Eq. [20(b) ]; therefore the propagator determined by our simulations for flow in porous media, designated $\mathbf{P}$, can be written as

$$
\mathbf{P}=\frac{4}{3} f_{P}(r) \mathbf{I}+\frac{4}{3} g_{P}(r)\left(\mathbf{r r} / r^{2}\right),
$$

where the velocity field due to a point force is given by replacing $\mathscr{J}$ with $P$ in Eq. (19). By determining $f_{P}(r)$ and $g_{P}(r)$, the porous medium propagator is fully specified. From our simulations we determine $\mathbf{M}_{\alpha \beta}^{P}$, whose off-diagonal component matrix relates the velocity of a field particle located at $\mathbf{x}_{\beta}$ to the force exerted by a source particle at $\mathbf{x}_{\alpha}$, and is therefore the porous medium propagator $\mathbf{P}$. If all the particles in the simulation are point forces, the off-diagonal component matrix of $\mathbf{M}_{\alpha \beta}^{P}$ gives the Green's function for flow in such a porous medium; on the other hand, if the particles are all finite-sized spheres, $\mathbf{M}_{\alpha \beta}^{P}$ relates the velocity of sphere $\beta$ to the force exerted by sphere $\alpha$.

The two functions $f_{P}(r)$ and $g_{P}(r)$ are determined by evaluating the velocity disturbance caused by a source particle as measured by the motion of the field particles. In the actual simulation, the following two summations, evaluated at discrete values of $r$, where $r$ is the distance from the source particle to the field particle, are performed:

$$
\begin{aligned}
(1-\phi) \frac{1}{m_{1}} \sum_{\beta=1}^{m_{1}} U_{1}^{\beta} & \sim \frac{1}{4 \pi r^{2}} \int_{\text {sphere }} u_{1} d S \\
= & {\left[f_{P}(r)+\frac{1}{3} g_{P}(r)\right] F_{1}^{\alpha}, } \\
(1-\phi) \frac{1}{m_{2}} \sum_{\beta=1}^{m_{2}} \mathbf{U}_{\beta} \cdot \mathbf{n} & \sim \frac{1}{2 \pi r^{2}} \int_{\text {hemisphere }} \mathbf{u} \cdot \mathbf{n} d S \\
& =\frac{1}{2}\left[f_{P}(r)+g_{P}(r)\right] F_{1}^{\alpha},
\end{aligned}
$$

where $m_{1}$ is the total number of particles contained within a spherical shell of thickness $\Delta r$ situated a distance $r$ from the source particle, and $m_{2}$ is the total number of particles contained within a hemispherical shell oriented such that its base is in the 2-3 plane. The subscript 1 refers to the 1 direction, $\mathbf{n}$ is the unit normal, and $\mathbf{U}^{\beta}$ is the velocity of particle $\beta$. The factors of $(1-\phi)$ correct for the fact that all field points sampled are considered fluid elements, i.e., the particle $\beta$ is assumed force-free in (15) in computing the summations in (22), while in reality a fraction $\phi$ are fixed in space and would have given zero to the sum. By evaluating each of these sums for imposed forces in the 1,2, and 3 directions and by considering each particle in turn as the source particle, a large body of statistical information is generated, yielding accurate estimates for $f_{P}(r)$ and $g_{P}(r)$.

The average force exerted by a fixed particle immersed in a uniform flow (nondimensionalized by $6 \pi \mu a U$ ), referred to as the drag coefficient $K^{-1}$, can be determined directly from Eq. (14). Specifically, the drag coefficient is given by

$$
K^{-1}=\frac{1}{3} \operatorname{tr}\left(\frac{1}{N} \sum_{\alpha=1}^{N} \sum_{\beta=1}^{N} \mathbf{R}_{\alpha \beta}^{*}\right),
$$

where $\mathbf{R}_{\alpha \beta}^{*}$, nondimensionalized by $6 \pi \mu a$, is as defined above. The dimensionless permeability $\alpha^{-2}$ is related to the drag coefficient by

$$
\alpha^{-2}=\frac{2}{9}(1 / \phi) K
$$

In the actual simulations, particles are placed randomly, via random sequential addition, in the periodic cell. Particles are not permitted to overlap. As mentioned above, it is important that the periodic cell be large enough that our results are not influenced by the imposition of periodic boundary conditions. In a Brinkman medium, the screening length, beyond which sphere interactions can be expected to differ significantly from those in a Stokes fluid, is the square root of the permeability, $\alpha^{-1}$. To ensure that the model system is indeed sufficiently large, we require that the ratio of the periodic cell size, $H$ [cf. Eq. (7)], to the Brinkman screening length be large compared to unity; e.g.,

$$
\alpha H \approx 3.4 N^{1 / 3} \phi^{1 / 6}>1 \text {. }
$$

In most of our simulations, $N=125 ; \phi$ varies from 0.002 $(\alpha H \approx 6)$ to $0.2(\alpha H \approx 13)$. Thus, as we shall see in Sec. III, our system is indeed large enough to display screening.

All of the simulations discussed in the next section were performed on a Cray X-MP supercomputer. In simulations involving the application of the Ewald summation technique, lattice sums are computed over 125 periodic cells, and approximately six minutes of CPU time are required. Over $90 \%$ of this time is spent computing the lattice sums; thus simulations of 125 particles without the application of the Ewald summation technique require less than $30 \mathrm{sec}$ of CPU time.

\section{RESULTS}

In this section we present simulation results for particle interactions in porous media, computed both with and without the application of the Ewald summation technique, and compare them with the Brinkman solution at four different values of $\phi: 0.01,0.002,0.05$, and 0.2 . The comparison is in terms of the functions $f(r)$ and $g(r)$ and the drag coefficient or resistivity $K^{-1}$. All distances are nondimensionalized by the sphere radius $a$. The results presented at a given value of $\phi$ are averaged over three independent, random realizations. In each realization every particle in turn is considered as the source particle, yielding a large body of statistical information. At given values of $\phi$ and $N$, several sets of simulations are generally performed to gauge the differences between results computed with different system specifications; e.g., finite-sized spheres versus point forces or $\langle\mathbf{u}\rangle \equiv 0$ vs $\langle\mathbf{F}\rangle \equiv 0$. 
In these cases, the same three realizations are used for each set of simulations. The variations between the results of the three realizations are generally quite small. In the $f_{P}(r)$ and $g_{P}(r)$ results, the standard deviations of the three-realization average are usually smaller than the size of the symbols on the figures by the third or fourth data point after $r=2$ (typically $r \approx 3.5$ to $r \approx 5$ ) and remain so for all larger values of $r$. For the first few data points, the standard deviation is generally about $10 \%$ of the average for both $f_{P}(r)$ and $g_{p}(r)$. More specific information concerning the variations between the three realizations will be cited only in those cases where the variations differ significantly from the general observations cited above.

We first consider results for $\phi=0.01$. Figures 1 (a) and 1 (b) display the simulation results, shown as $X$ 's and + 's, for $f_{P}(r)$ and $g_{P}(r)$ for a system of 125 point forces. In this and all subsequent figures the $X$ 's correspond to simulations of point forces for which $\langle u\rangle \equiv 0$ and the + 's to simulations of point forces for which $\langle F\rangle \equiv 0$. The results in Figs. 1(a) and $1(b)$ are for simulations performed with the application of the Ewald summation technique. The solid lines are the Brinkman medium results, given by Eqs. (20), with $\alpha$ as given by the infinite dilution result Eq. (20c), and the dashed lines are pure fluid (Stokes propagator) results;
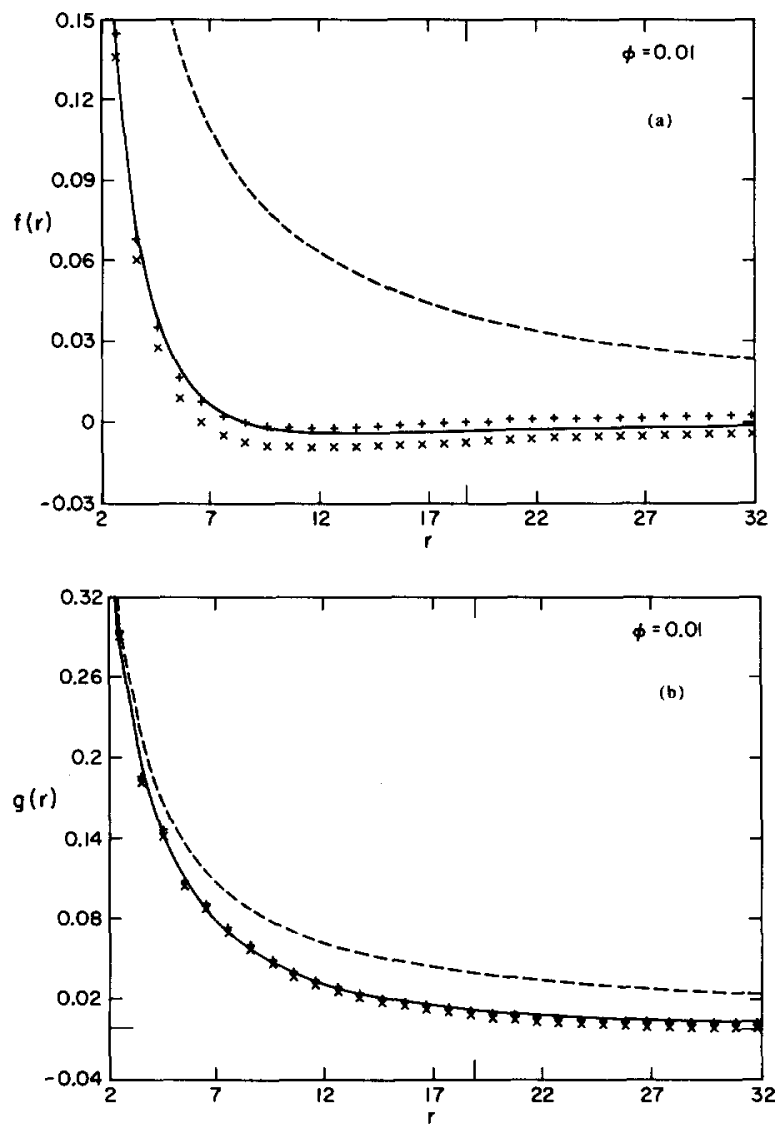

FIG. 1. Comparison of $\phi=0.01$ theoretical and simulation results for (a) $f(r)$ and $(b) g(r)$. Simulations performed for 125 point forces with the application of the Ewald summation technique. The $X$ 's are $\langle u\rangle \equiv 0$ results; + 's are $\langle\mathbf{F}\rangle \equiv 0$ results, the solid curves are the Brinkman propagator $f_{\mathrm{B}}(r)$ and $g_{\mathrm{B}}(r)$ functions, and the dashed curves are the Stokes propagator. The vertical line on the abscissa in these and all subsequent figures indicates the half-box width.
$f_{S}(r)=g_{S}(r)=\left(\frac{3}{4}\right) r^{-1}$. The half-box width $(H / 2=18.7)$ is indicated by a vertical line on the abscissa. Both sets of simulation results agree quite well with the Brinkman medium results and deviate from one another only slightly and by a nearly uniform offset. As discussed in Sec. II, the two sets of simulation results deviate from one another by an $O(1 / N)$ amount; a larger variation between them will be evident in the $N=27$ results shown below.

Note that the simulation results agree with the Brinkman propagator over the entire range of $r$, up to $r \approx 32$. At the parameter values for these simulations $(\phi=0.01, N$ $=125$ ) the box size $H$ is 37.4. The maximum distance between two particles is $\sqrt{3} H / 2(=32.4)$, the distance from the center of the periodic cell to a corner. Because portions of spherical surfaces of radius $r$ extend beyond the periodic cell (a cube of side $H$ ), for values of $r$ beyond $H / 2$, the summations in Eq. (19) are not actually over the surfaces of spheres of radius $r$ but over the portion of the surface of a sphere of radius $r$ that falls within a cube of side $H$. This geometrical limitation results in a diminution in the amount of statistical information as $r \rightarrow \sqrt{3} H / 2$, but does not affect the validity of Eq. (19) or the agreement between the simulation results and the Brinkman equation in the region $H / 2<r<\sqrt{3} H / 2$.

Because the Brinkman equation is rigorously valid for a random, infinite system as $\phi \rightarrow 0$, we expect our simulation
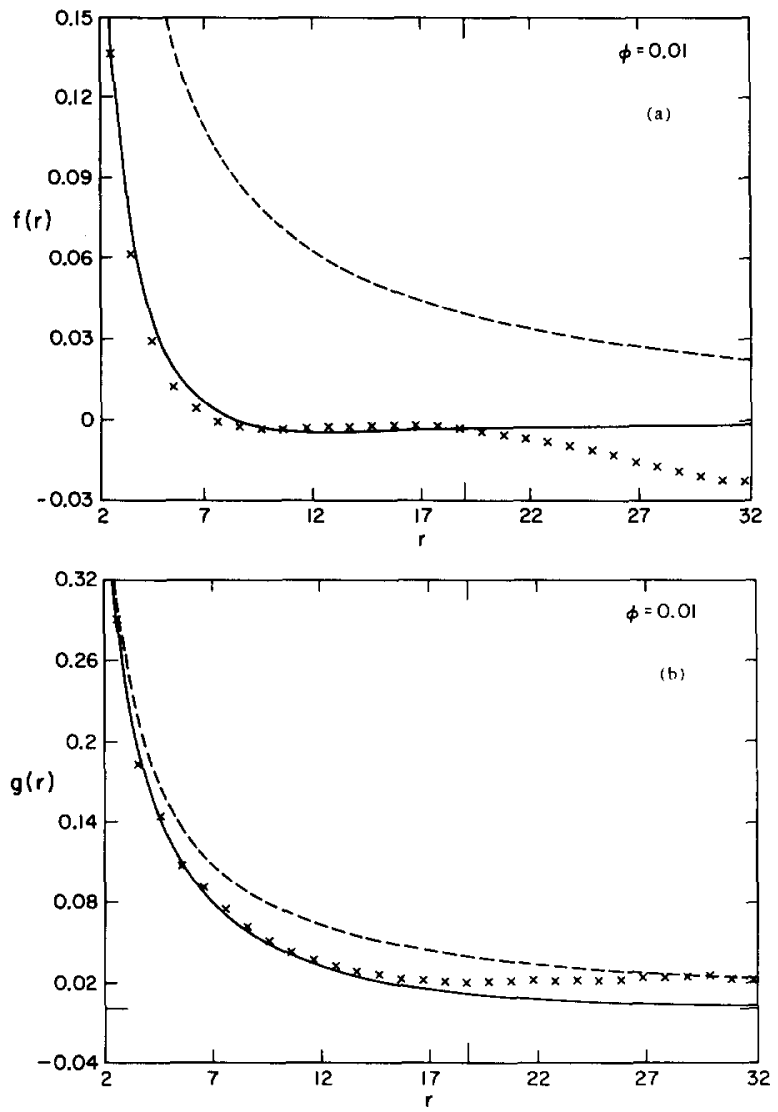

FIG. 2. Comparison of $\phi=0.01$ theoretical and simulation results for (a) $f(r)$ and $(b) g(r)$. Simulations performed for 125 point forces without the application of the Ewald summation technique. The $X$ 's are $\langle\mathbf{n}\rangle \equiv 0$ simulation results, the solid curves are the Brinkman propagator, and the dashed curves are the Stokes propagator. Note the deviation of the simulation and Brinkman results for $r>H / 2$. 
results to recover the Brinkman propagator at low $\phi$ for sufficiently large $N$. Figures 1 (a) and 1 (b) indicate that a periodically replicated random system of 125 point forces does indeed behave as a Brinkman medium when the particles are specified to be fixed in space. This can be taken as verification that the inversion of the mobility matrix $\mathbf{M}^{*}$ results in all many-body scatterings (at least at the level of point forces), yielding a medium that behaves fundamentally differently than either a pure fluid or a suspension of force-free particles.

We next present results for simulations of point forces at the same parameter values as above ( $\phi=0.01, N=125$ ), but without the application of the Ewald summation technique. In these simulations, periodic boundary conditions are imposed but no lattice sums are performed; i.e., particles interact only with their nearest neighbors and not with an infinite replication of images. The results for $f_{P}(r)$ and $g_{P}(r)$ are shown in Figs. 2(a) and 2(b). Again the $X$ 's correspond to point-force simulation results with $\langle\mathbf{u}\rangle \equiv 0$, the solid lines to the Brinkman propagator and the dashed line to the Stokes propagator. Though agreement with the Brinkman solution is good for both functions in the range $2<r<17$, agreement beyond $r \approx 20$ becomes poor, particularly for $g_{P}(r)$, which tends to the Stokes solution for $r>27$. Comparison of Figs. 2(a) and 2(b) with Figs. 1(a) and 1(b) clearly illustrates the importance of the effect of distant particles, even for $\phi$ as low as 0.01 . As will be seen below, the effect of distant particles is similarly important for $\phi=0.05$.

Having established that the $N=125$ point-force simulations at $\phi=0.01$ with the application of Ewald sums do indeed reproduce Brinkman's result, the effect of the size of the periodic cell will now be considered. Shown in Figs. 3(a) and $3(\mathrm{~b})$ are $f_{P}(r)$ and $g_{P}(r)$ for systems of 27 point forces for $\phi=0.01(H / 2=11.2)$ for simulations performed with Ewald sums. Again, the $X$ 's correspond to $\langle\mathbf{u}\rangle \equiv 0$ and the + 's to $\langle\mathbf{F}\rangle \equiv 0$. The standard deviations in the $f_{P}(r)$ and $g_{P}(r)$ data points for the $N=27$ simulation results are slightly higher than those observed with $N=125$. In $g_{P}(r)$, standard deviations of $O(0.01$ to 0.02$)$, for both the $\langle\mathbf{u}\rangle \equiv 0$ and $\langle\mathbf{F}\rangle \equiv 0$ simulations, exist in the second, third, and sixth data points $(r=3.45,4.42,7.33)$. At larger values of $r$, the standard deviations for the $N=27$ simulation $f_{P}(r)$ and $g_{P}(r)$ functions are, though small [ $\sim O(0.001)$, smaller than the size of the symbols], about a factor of 3 larger than those for the $N=125$ simulation functions.

It is apparent from Figs. 3(a) and 3(b) that, though the trends agree with the Brinkman result, the offsets between the simulation and Brinkman results and between the two simulation results are noticeably greater than for the $N$ $=125$ simulations [compare Figs. 1(a) and 1(b)]. Further, these variations are statistically significant. As shown in Sec. II the two sets of simulation results deviate from one another by an $O(1 / N)$ amount. To determine the variation of the offset between the Brinkman and simulation results with system size, additional simulations with $\langle\mathbf{u}\rangle \equiv 0$ were performed at $\phi=0.01$ for systems of 64 and 90 point forces. The offset is quantified by computing the average of the difference between the simulation and Brinkman results over the range $H / 2<r<\sqrt{3} H / 2$. Least squares fits for log-
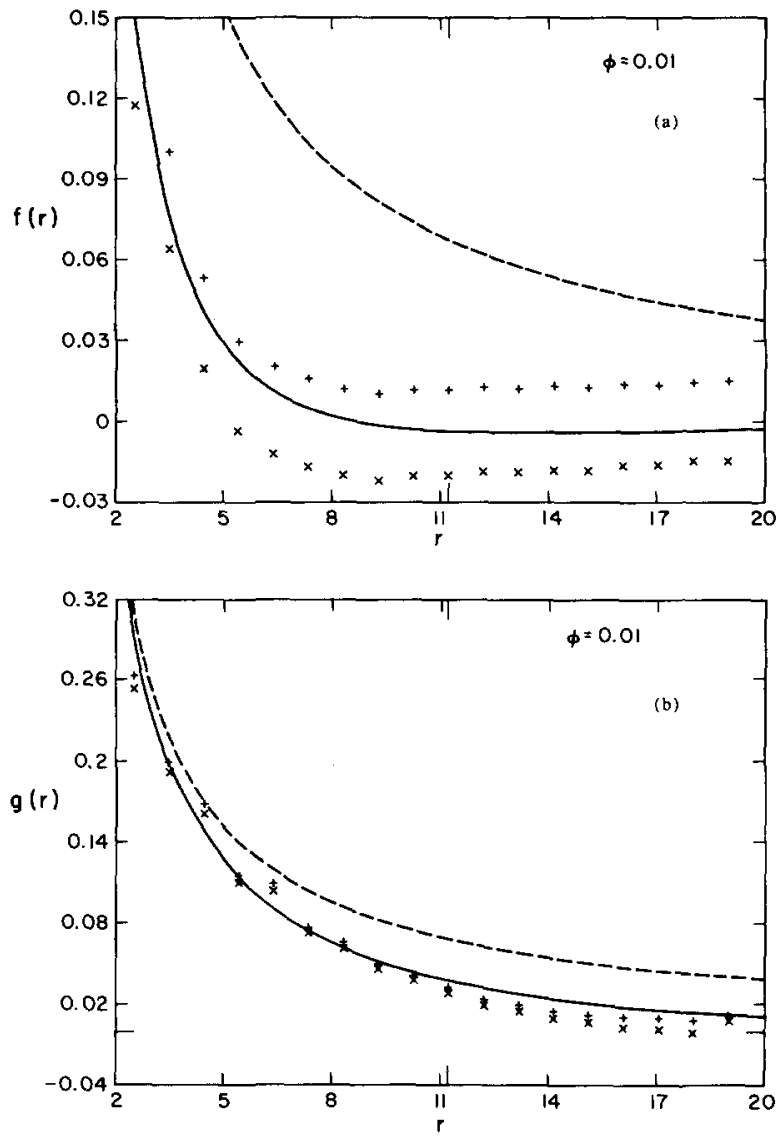

FIG. 3. Comparison of $\phi=0.01$ theoretical and simulation results for (a) $f(r)$ and (b) $g(r)$. Simulations performed for 27 point forces with Ewald sums. The $X$ 's are $\langle u\rangle \equiv 0$ simulation results, the + 's are $\langle\mathbf{F}\rangle \equiv 0$ simulation results, the solid curves are the Brinkman propagator, and the dashed curves are the Stokes propagator. The deviations between the two simulation results for $f(r)$ and between the simulation results and the Brinkman function are noticeably larger than for the $N=125$ simulations [see Fig. 1 (a) ].

$\log$ plots of offset in both $f_{P}(r)$ and $g_{P}(r)$ vs $N$ for $27 \leqslant N \leqslant 125$ give slopes of $-0.94 \pm 0.05$ and -0.77 \pm 0.03 , respectively. Thus over this range of $N$ the average offset scales approximately as $N^{-0.86}$. Only considering the range $64 \leqslant N \leqslant 125$, the slopes are $-1.03 \pm 0.13$ and $-0.87 \pm 0.01$ for the offsets in $f_{P}(r)$ and $g_{P}(r)$, respectively, giving an average offset that scales approximately as $N^{-0.95}$. Therefore, it appears that the difference between the Brinkman and simulation results scales approximately as $N^{-1}$, as does the offset between the two sets of simulation results. Further, it is evident from Figs. 1(a) and 3(a) that for simulations with $\langle\mathbf{F}\rangle \equiv 0, f_{P}(r)$ approaches $f_{\mathrm{B}}(r)$ from above and for simulations with $\langle\mathbf{u}\rangle \equiv 0$ the approach is from below. Such a trend does not exist for the $g_{P}(r)$ functions.

We next briefly consider simulation results for very dilute systems: $\phi=0.002$. These simulations, performed for 125 point forces with Ewald sums and $\langle u\rangle \equiv 0$, are of interest because they allow comparison over a large range of $r(H /$ $2=32.0$ ). Figures $4(\mathrm{a})$ and $4(\mathrm{~b})$ display the results for $f_{P}(r)$ and $g_{P}(r)$; agreement with the Brinkman solution is excellent over the entire range of the results, $r<54$. Note the only slight difference between the Stokes and Brinkman $g(r)$ functions in Fig. 4(b) and the agreement of the simulation 

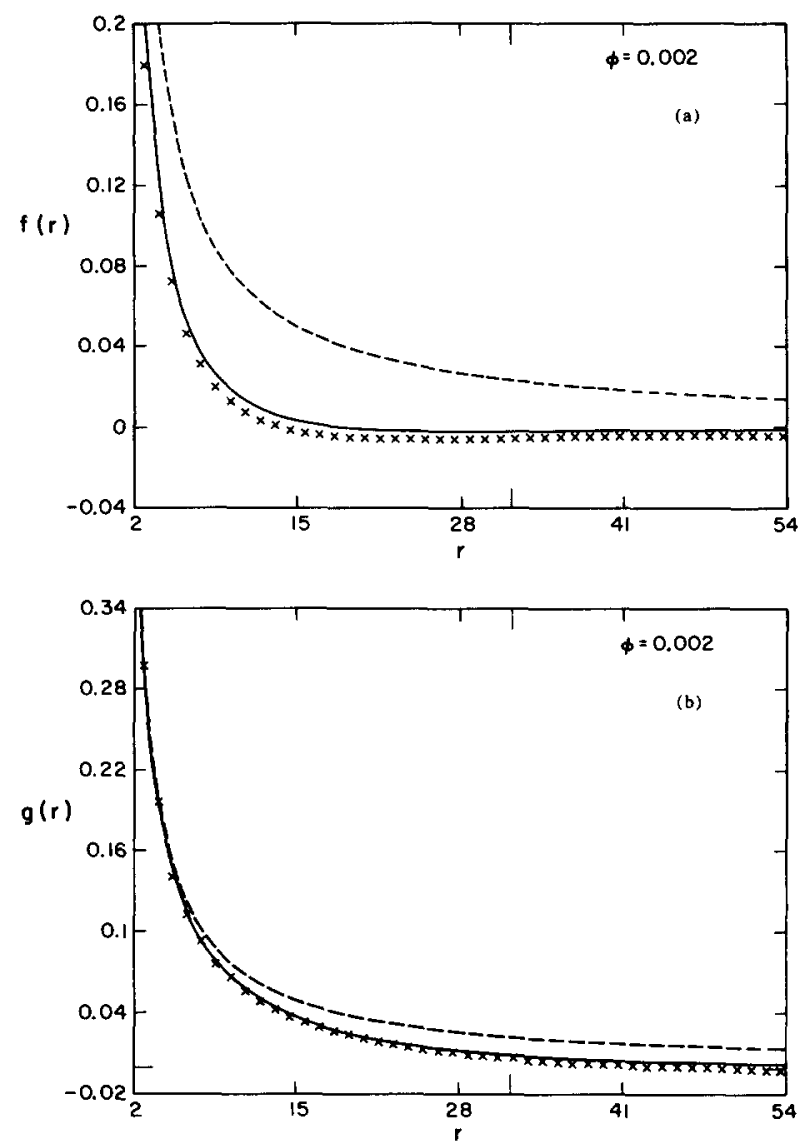

FIG. 4. Comparison of $\phi=0.002$ theoretical and simulation results for (a) $f(r)$ and (b) $g(r)$. Simulations performed for 125 point forces with Ewald sums. The $\times$ 's are $\langle u\rangle \equiv 0$ simulation results, the solid curves are the Brinkman propagator, and the dashed curves are the Stokes propagator.

results with the latter. Despite the only slight quantitative differences between the simulation (or Brinkman) results and the pure fluid (Stokes) results, it is important to emphasize that the simulation and Brinkman $f(r)$ and $g(r)$ functions decay as $1 /\left(\alpha^{2} r^{3}\right)$ at large $r$ while the pure fluid functions decay as $1 / r$. This fundamental difference can be readily appreciated if one computes the spherical average of the Brinkman and Stokes propagators: the Brinkman propagator behaves as $e^{-\alpha r} / r$, while the Stokes propagator still only decays as $1 / r$.

We now turn from very dilute systems to a consideration of moderately dilute porous media: $\phi=0.05$. The simulation results presented at $\phi=0.05$ are both for systems of 125 point forces and 125 identical finite-sized spheres. When considering systems of finite-sized spheres, comparison of the simulation results with the Green's function for the Brinkman equation may no longer be appropriate. Rather, the simulation results should be compared to the Brinkman equation equivalent of the Rotne-Prager tensor for Stokes flow; i.e., the Brinkman Green's function plus twice the Faxén law contribution, ${ }^{8}$

$$
\begin{aligned}
& \mathscr{J}^{(1)}=\left\{1+\left[\frac{2 B_{2}}{B_{0}}+\left(\frac{\alpha B_{2}}{B_{0}}\right)^{2}\right] \nabla^{2}\right\} \mathscr{J}, \\
& B_{0}(\alpha)=1+\alpha+\frac{1}{3} \alpha^{2} \\
& B_{2}(\alpha)=\left(e^{\alpha}-B_{0}\right) / \alpha^{2},
\end{aligned}
$$

where $\mathscr{J}$ is given by Eq. (20a). The $\left(\alpha B_{2} / B_{0}\right)^{2} \nabla^{2} \mathscr{J}$ term in (26a) arises because $\nabla^{2} \mathscr{J}$ satisfies the Helmholtz equation, giving $\nabla^{4} \mathscr{J}=\alpha^{2} \nabla^{2} \mathscr{J} .^{18}$ Note that $\nabla^{2} \mathscr{J}$ decays exponentially with $r$, so its effect is only noticeable at small $r$. Further, the dilute limit approximation for $\alpha$, Eq. $(20 \mathrm{c})$, will not be used but rather the value for $\alpha$ obtained from the simulation [cf. Eq. (24)].

The results for $f_{P}(r)$ and $g_{P}(r)$ for simulations performed with Ewald sums are displayed in Figs. 5(a) and $5(b)$. The $X$ 's correspond to point force, $\langle u\rangle \equiv 0$ simulation results; the + 's to point force, $\langle\mathbf{F}\rangle \equiv 0$ simulation results; $O$ 's to finite sized sphere, $\langle\mathbf{u}\rangle \equiv 0$ simulation results; the solid curves to the Brinkman propagator $f_{\mathrm{B}}(r)$ and $g_{\mathrm{B}}(r)$ functions computed using the point force simulation result for $\alpha$ $\left(\alpha_{p_{f}}=0.6775\right)$, and the broken curves to the $f_{\mathrm{B}}(r)$ and $g_{\mathrm{B}}(r)$ functions, corresponding to $\mathscr{J}^{(1)}$ in Eq. (26), computed using the finite-sized sphere simulation result for $\alpha$ $\left(\alpha_{f_{s}}=0.6348\right)$. By $r \approx 6$, the solid and broken curves for both $f_{\mathrm{B}}(r)$ and $g_{\mathrm{B}}(r)$ are in good agreement despite the slightly different $\alpha$ values.
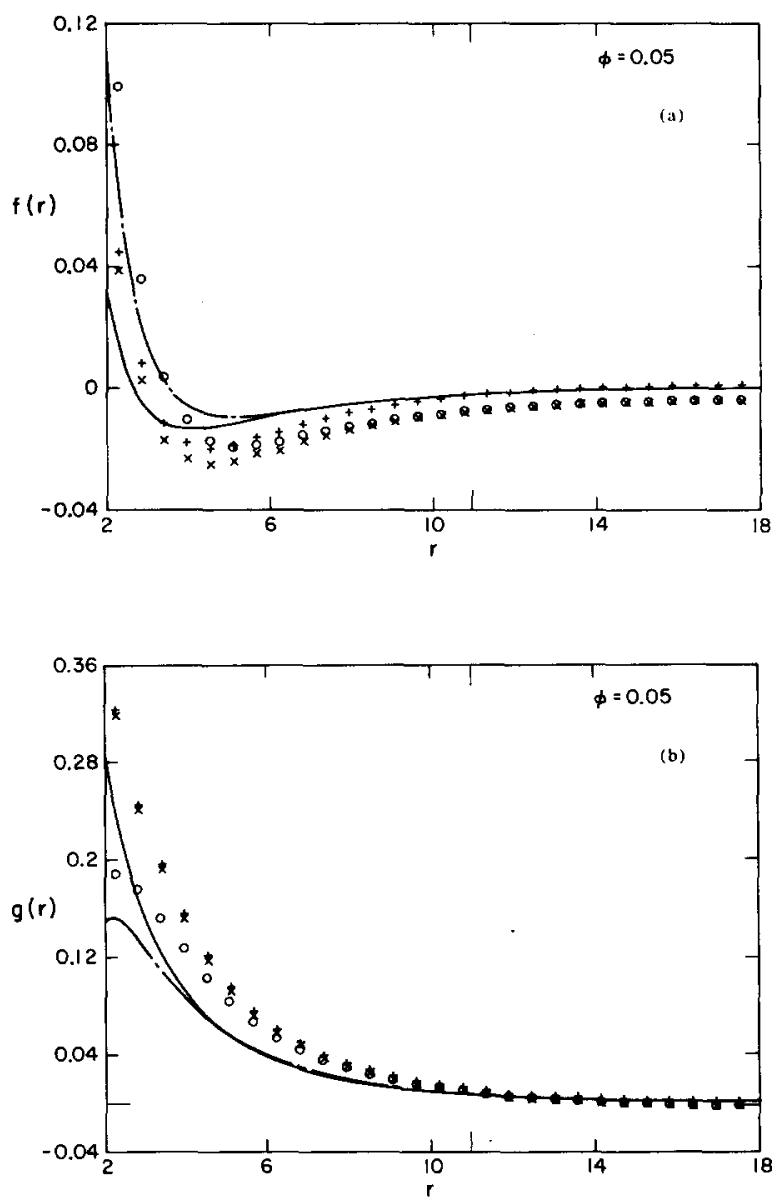

FIG. 5. Comparison of $\phi=0.05$ theoretical and simulation results for (a) $f(r)$ and $(b) g(r)$. Simulations performed for 125 particles with the application of Ewald sums. The $X$ 's are $\langle u\rangle \equiv 0$ point force simulation results, the + 's are $\langle\mathbf{F}\rangle \equiv 0$ point force simulation results, the $O$ 's are $\langle u\rangle \equiv 0$ simula. tion results for finite-sized spheres, the solid curves are Brinkman propagator functions ( $\alpha=0.6775$ ), and the broken curves are Brinkman RotnePrager functions ( $\alpha=0.6348)$. The deviations between the simulation and Brinkman results indicates the loss of accuracy of the Brinkman equation at $\phi=0.05$. 
Though quantitative agreement between the Brinkman functions and the simulation results does not exist except at large values of $r$, qualitatively the results are in fair agreement even at moderate values of $r$. Both the simulation and Brinkman results, for both point forces and finite-sized spheres, display negative minimums in $f(r)$ at moderate values of $r$. The $f_{P}(r)$ results for systems of finite-sized spheres lie above those for systems of point forces, just as the $f_{\mathbf{B}}(r)$ functions of Eq. (26) lies above the Brinkman propagator $f_{\mathrm{B}}(r)$ function. In the $g(r)$ functions both the simulation results for systems of finite-sized spheres and the Brinkman Rotne-Prager result level off near $r=2$ while the simulation results for systems of point forces and the Brinkman propagator decrease monotonically. In all cases, however, quantitative differences are apparent between the simulation and Brinkman results.

It is our belief that the discrepancies between the simulation and Brinkman results at $\phi=0.05$ are real and will persist as $N \rightarrow \infty$. The small deviations between the $\langle u\rangle \equiv 0$ and $\langle F\rangle \equiv 0$ point force results indicate that the simulation results have essentially converged for increasing $N$, and considerable quantitative differences still exist between the simulation and Brinkman results, particularly in $g(r)$. Further, simulations performed with systems of 27 point forces do not indicate that the simulation results tend toward the Brinkman results with increasing $N$. For simulations in which $\langle\mathbf{F}\rangle \equiv 0$, the $N=27 f_{P}(r)$ results lie consistently above the $N=125 f_{P}(r)$ results. At $r=2.28$, where the largest absolute deviation for $r \leqslant 10$ between the two occurs $(\sim 0.01)$, the discrepancy in the $N=27$ results relative to the $N=125$ results is $16 \%$. The fact that the minimum in $f_{P}(r)$ for $N=27$ simulations lies above that for $N=125$ simulations suggests that the minimum becomes more negative, and thus deviates more from the Brinkman result, as $N$ increases. The $g_{P}(r)$ results for the two simulations deviate only slightly; at $r=2.28$ the $N=27$ simulation result exceeds that of the $N=125$ simulation result by $1.5 \%$, while at $r=3.82$ the $N=27$ result falls below the $N=125$ result by $6 \%$. Thus, there is no evidence that the $\phi=0.05$ point force simulation results tend to the Brinkman solution with increasing $N$.

The Brinkman equation provides an exact description of flow in random porous media as $\phi \rightarrow 0$. Rigorous derivation of the Brinkman equation requires only point force interactions among the fixed particles-no higher moments are necessary. Therefore, the quantitative differences between the point force simulation results and the Brinkman propagator at $\phi=0.05$ are significant; they indicate that the Brinkman equation has begun to lose accuracy for systems of point forces at $\phi$ as low as 0.05 .

The next simulation results presented are for $\phi=0.05$, $N=125$ with $\langle u\rangle \equiv 0$ but without the application of the Ewald summation technique. Simulations of systems of point forces under these conditions resulted in nonpositive definite mobility matrices for some realizations, while simulations of systems of finite-sized spheres yielded well-behaved mobility matrices in all cases. Results for $f_{P}(r)$ and $g_{P}(r)$ for finite sized spheres are shown in Figs. 6(a) and 6(b). The open circles are the simulation results and the solid and broken curves are the Brinkman propagator and
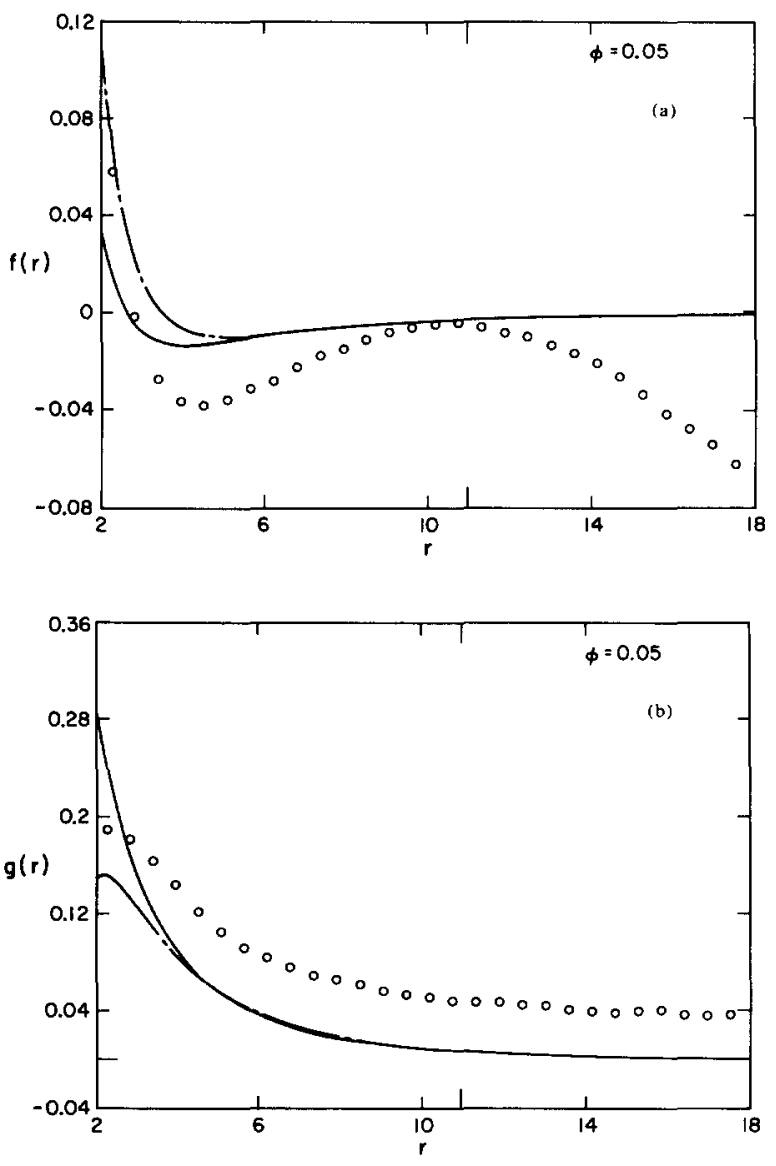

FIG. 6. Comparison of $\phi=0.05$ theoretical and simulation results for (a) $f(r)$ and $(b) g(r)$. Simulations performed for 125 finite-sized spheres without Ewald sums. The $O$ 's are $\langle u\rangle \equiv 0$ simulation results, the solid curves are Brinkman propagator functions $(\alpha=0.6775)$, and the broken curves are Brinkman Rotne-Prager functions $(\alpha=0.6348)$. The $f(r)$ simulation results are aphysical for $r>H / 2$.

Brinkman Rotne-Prager results, respectively, with the same $\alpha$ 's as in Figs. 5(a) and 5(b). (As discussed below, simulations performed without the application of the Ewald sum technique do not yield reasonable $\alpha$ values. ) The results for $f_{P}(r)$ agree qualitatively with the Ewald summed $\phi=0.05$ simulation results [Fig. 5(a)] for $2<r<10$, but beyond this the trend is entirely different. For $r>H / 2$, the $f_{P}(r)$ simulation results are rather aphysical; they increase in magnitude with increasing separation. The $g_{P}(r)$ results appear more reasonable, though they deviate considerably from the results of Fig. 5(b) and actually tend more toward the Stokes solution [not shown in Fig. 6(b)] than the Brinkman solution at large $r$. This was also the case for the $\phi=0.01 g_{P}(r)$ results for simulations performed without Ewald sums [see Fig. 2(b) ].

The last set of simulation results to be considered is for moderately concentrated suspensions, $\phi=0.2$. In simulations with $N=125$, the mobility matrix lost positive definiteness in all cases when the Ewald summation technique was not applied (for systems of either point forces or finitesized spheres) and also when the Ewald summation technique was applied to systems of point forces. Thus the only results presented are for systems of 125 finite-sized spheres simulated with Ewald sums. The $f_{P}(r)$ and $g_{P}(r)$ results for 
these simulations are shown in Figs. 7 (a) and 7(b). The open circles correspond to the $\langle u\rangle \equiv 0$ simulations, the asterisks to $\langle F\rangle \equiv 0$ simulation results, the solid curves to the Brinkman propagator using the value for $\alpha$ obtained from the simulation $(\alpha=1.989)$, and the broken curves to the Brinkman Rotne-Prager functions using the same value for $\alpha$. The standard deviations in the $f_{P}(r)$ and $g_{P}(r)$ simulation results are very small for all values of $r$-in all cases smaller than the size of the symbols. The simulation and Brinkman results deviate significantly, though the trends are still in qualitative agreement. Note that the deviations between the two are similar to those observed at $\phi=0.05$; the $f_{P}(r)$ simulation results display a more negative minimum than do the $f_{\mathrm{B}}(r)$ curves, while the $g_{P}(r)$ simulation results lie considerably above the $g_{B}(r)$ curves at small to moderate values of $r$.

The $\phi=0.2$ simulation results should be interpreted more as an indication of the loss of the validity of the Brinkman equation than as an accurate estimate of the actual porous media $f(r)$ and $g(r)$ functions. At $\phi=0.2$, characteristic nearest-neighbor interparticle spacings $r_{12}\left[r_{12}=\left(\frac{4}{3} \pi /\right.\right.$ $\left.\phi)^{1 / 3}\right]$ are 2.76 sphere radii, to be contrasted with $7.48,12.8$, and 4.38 at $\phi$ values of $0.01,0.002$, and 0.05 , respectively. The relatively near spacing of the spheres at $\phi=0.2$ indicates that higher moments in the expansion of the integral
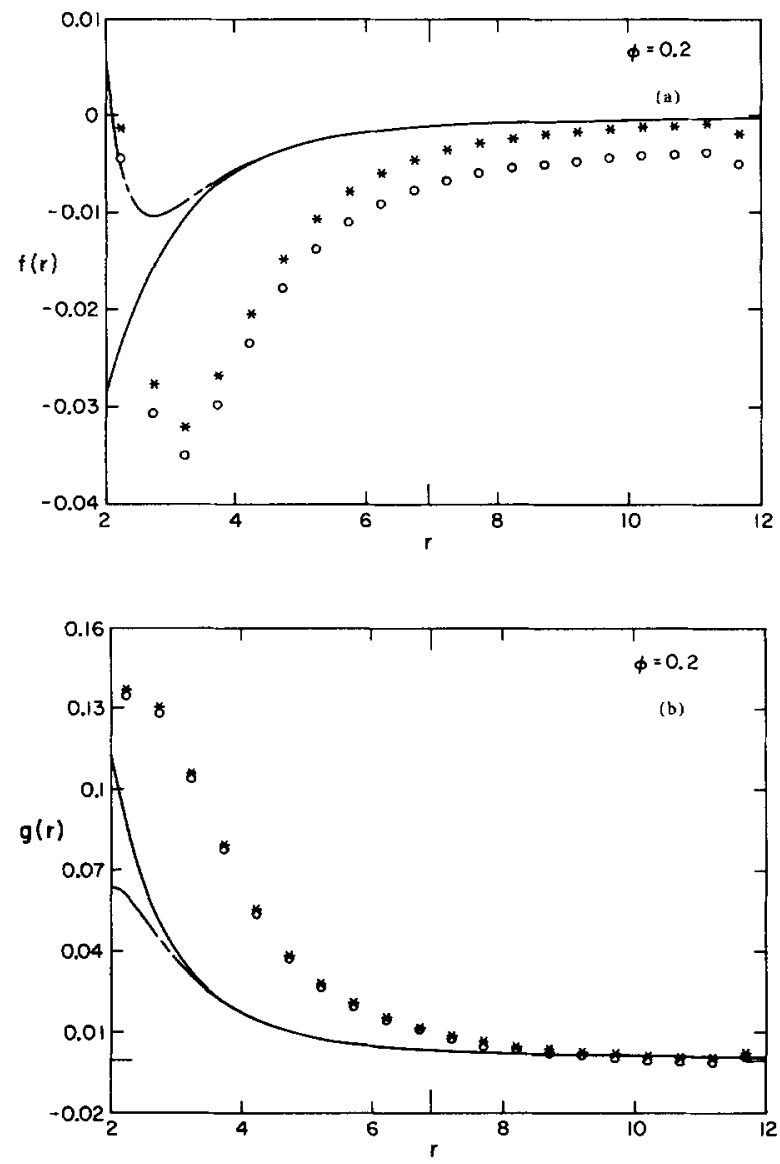

FIG. 7. Comparison of $\phi=0.2$ theoretical and simulation results for (a) $f(r)$ and (b) $g(r)$. Simulations performed for 125 finite-sized spheres with Ewald sums. The $O$ 's are $\langle u\rangle \equiv 0$ simulation results, the ${ }^{*}$ 's are $\langle\mathbf{F}\rangle \equiv 0$ simulation results, the solid curves are Brinkman propagator functions $(\alpha=1.989)$, and the broken curves are Brinkman Rotne-Prager functions $(\alpha=1.989)$. The Brinkman equation is not valid at $\phi=0.2$. expression for the Stokes flow velocity field may be required; in other words, the Rotne-Prager tensor may no longer suffice as a description of two-sphere mobility interactions. Further, lubrication may need to be introduced into the resistance matrix to accurately account for very short-ranged interactions. These extensions are available and straightforward, ${ }^{14,15}$ but require considerably more computation time than the present method and were therefore not implemented. Also, the use of bed particles rather than infinitesimal test particles may affect the behavior near $r=2$ at this high value of $\phi$. The $\phi=0.2$ simulation results may find additional use as a "known" result to serve as a comparison with a theoretical treatment of nondilute porous media where sphere-sphere interactions are approximated by the RotnePrager tensor.

Finally, we consider the results for the drag coefficient $K^{-1}$, for the simulations discussed above. Reasonable results for the drag coefficient are obtained only in simulations performed with the application of Ewald sums. Without Ewald sums, for 125 particles, $K^{-1}=0.114$ at $\phi=0.01$ for point forces and $K^{-1}=0.0712$ at $\phi=0.05$ for finite-sized spheres. These results are clearly unrealistic; $K^{-1}$ must exceed unity and increase with increasing $\phi$.

Displayed in Table I are drag coefficient results with simulations performed with Ewald sums. The standard deviations presented correspond to variations between the averages for each of the three distinct realizations. In the last column are the results of Kim and Russel ${ }^{13}$; for $\phi \leqslant 0.05$ their low $\phi$ expansion result [their Eq. (2.14)] is presented and for $\phi=0.2$ their numerical value. Several trends are apparent from the simulation results. Most importantly, $K^{-1}$ increases with increasing $\phi$; this is not the case when the Ewald summation technique is not applied. Values for $K^{-1}$ are consistently higher for simulations of systems of point forces than for simulations of systems of finite-sized spheres, presumably a result of the slightly different nature of the manybody reflections that occur upon inversion of the mobility matrix for a system of finite-sized spheres compared to those for a system of point forces.

The $K^{-1}$ values presented in Table I increase with decreasing system size, although this trend does not appear to be statistically significant; i.e., the standard deviation also increases with decreasing system size, as would be expected.

TABLE I. Results for the drag coefficient $K^{-1}$ defined in Eq. (24). All simulations were performed with the application of the Ewald summation technique.

\begin{tabular}{|c|c|c|c|c|}
\hline$\phi$ & $N$ & $\begin{array}{c}\text { Point force } \\
\text { or } \\
\text { finite size }\end{array}$ & $K^{-1}$ & $\left(K^{-1}\right)^{a}$ \\
\hline 0.002 & 125 & PF & $1.098 \pm 0.024$ & 1.102 \\
\hline 0.01 & 125 & $\mathbf{P F}$ & $1.259 \pm 0.044$ & 1.280 \\
\hline 0.01 & 125 & FS & $1.237 \pm 0.043$ & 1.280 \\
\hline 0.01 & 27 & $\mathbf{P F}$ & $1.320 \pm 0.082$ & 1.280 \\
\hline 0.05 & 125 & $\mathbf{P F}$ & $2.040 \pm 0.032$ & 1.981 \\
\hline 0.05 & 125 & FS & $1.791 \pm 0.035$ & 1.981 \\
\hline 0.05 & 27 & PF & $2.178 \pm 0.126$ & 1.981 \\
\hline 0.2 & 125 & FS & $4.396 \pm 0.056$ & 4.61 \\
\hline
\end{tabular}

"Results from Kim and Russel. ${ }^{13}$ 
For very small systems, however, a statistically significant increase in the drag coefficient would be expected to occur. Due to the imposition of periodic boundary conditions, a small system is not truly random and would behave somewhat as an ordered, periodic system. Reference to Kim and Russe $^{13}$ (their Table III) shows that the drag coefficient is consistently greater (at least for $\phi \leqslant 0.45$ ) for ordered as compared to random systems.

Finally, we note that the agreement between the simulation results for the drag coefficient and the results of Kim and Russel is quite good; within $10 \%$ in all cases. This would be expected for low $\phi$, but it is somewhat surprising that the agreement persists for $\phi$ as large as 0.2 . At $\phi=0.2$, the Brinkman equation does not provide a quantitative basis for computing sphere interactions in a porous medium, as is evident from Figs. 7(a) and 7(b). Although the $\phi=0.2$ simulation results for $f_{P}(r)$ and $g_{P}(r)$ are only approximate (see discussion above), the drag coefficient result is expected to be more reliable, due to the relatively small effect of lubrication in the calculation of the drag coefficient (refer to Brady et al. ${ }^{15}$ for a discussion of this point). In their permeability calculations, however, Kim and Russel approximate sphere-sphere interactions as via a Brinkman medium at all values of $\phi$. Though this approximation loses accuracy at moderate and high $\phi$, there are apparently compensating effects in their calculations that render the final result for the permeability accurate.

\section{CONCLUSIONS}

In this paper, we have applied the general methodology of Stokesian dynamics to determine the form of the fundamental solution for flow in porous media. In simulations of dilute porous media, the results clearly show that the system behaves as a Brinkman medium, with long-ranged interactions screened by intervening fixed particles, rather than as a viscous fluid. This "effective medium" behavior of the simulated porous medium comes about upon the inversion of a properly constructed $N$-particle mobility matrix, which itself derives from a moment expansion of the integral representation of the Stokes velocity field. Thus the effective properties of the medium arise naturally out of the Stokesian dynamics methodology; they need not be postulated a priori. Indeed, we have presented our analysis of the Brinkman propagator as if it were obvious that Stokesian dynamics necessarily gives the correct answer. In retrospect, it is obvious that Stokesian dynamics is correct, and we hope the present study provides a convincing proof. In a subsequent publication, we shall show that the effective interactions among particles in sedimenting suspensions can be determined in much the same way as those of porous media using the Stokesian dynamics method. ${ }^{19}$

The results presented in Sec. III demonstrate the agreement between the simulation and the Brinkman equation at low $\phi$, but quantitative differences between the two, indicating the loss of validity of the Brinkman equation, are evident for $\phi \geqslant 0.05$. The results also show the importance of including the effects of distant particles via the Ewald summation technique; at $\phi=0.01$ and $\phi=0.05$ qualitative inaccuracies appear at large distances from the source particle in simulations performed without Ewald sums. Over more restricted regions $(r<H / 2)$, however, the simulations performed without the application of Ewald sums do provide reasonable results. It may seem surprising that the Brinkman equation starts to break down at what appears to be a rather low volume fraction, but it should be realized that a characteristic interparticle spacing at $\phi=0.05$ is only slightly larger than four particle radii; particles are actually rather close together. Permeability or drag coefficient calculations only yield realistic values when the Ewald summation technique is applied; in these cases agreement with the self-consistent results of Brinkman ${ }^{5}$ and the results of Kim and Russel ${ }^{13}$ is consistently good up to $\phi=0.2$, the highest value of $\phi$ considered.

In addition to presenting the form of the fundamental solution for flow in porous media at various values of $\phi$, the simulation results at $\phi=0.05$ and $\phi=0.2$ may find additional use as a basis of comparison for future theoretical work. Rubinstein ${ }^{12}$ rigorously derived the Brinkman equation by considering a dilute system of fixed spheres approximated as point forces, but his diluteness criterion is highly restrictive. In another paper, Rubenstein ${ }^{20}$ suggests that subsequent theoretical approaches, aimed at rigorously describing porous media at higher values of $\phi$, may involve higher multipoles. In this case, the theoretical results may be directly comparable to our simulation results for systems of finitesized spheres.

The procedure presented in this paper is appropriate for studying at most moderately concentrated suspensions. Extensions to more concentrated systems are developed and applied to the study of bulk properties (the nature of the fundamental solution is not considered) of ordered systems by Brady et $a l .^{15}$; disordered systems are presently under study. For disordered systems, a Monte Carlo method is applied to assure that hard-sphere distributions are obtained; the simple random sequential addition used in the present study reaches a percolation threshold at moderate values of volume fraction and subsequently fails. Further, at high volume fractions the full Stokesian dynamics method, which includes lubrication interactions, must be used.

\section{ACKNOWLEDGMENTS}

We wish to thank J. Lester and R. Phillips for their assistance with some of the computer programming and $\mathrm{G}$. Bossis for many useful discussions.

Computer time was provided on the San Diego Supercomputer Center Cray X-MP through a National Science Foundation allocation. Partial support for this work was through National Science Foundation Grant No. CBT8696067.

${ }^{1}$ H. P. G. Darcy, Les fontanes publiques de la ville de Dijon (Dalmont, Paris, 1856).

${ }^{2} \mathrm{~J}$. B. Keller, in Statistical Mechanics and Statistical Methods in Theory and Applications, edited by U. Landman (Plenum, New York, 1977), pp. 631644. 
${ }^{3} \mathrm{~S}$. Whitaker, Transport in Porous Media 1, 3 (1986).

${ }^{4}$ H. Brenner, Philos. Trans. R. Soc. London Ser. A 297,81 (1980); P. M. Adler and H. Brenner, Phys. Chem. Hydrodyn. 5, 245 (1984).

${ }^{5}$ H. C. Brinkman, Appl. Sci. Res. A 1, 27 (1947).

${ }^{6}$ C. K. W. Tam, J. Fluid Mech. 38, 537 (1969).

${ }^{7}$ S. Childress, J. Chem. Phys. 56, 2527 (1972).

${ }^{8}$ I. D. Howells, J. Fluid Mech. 64, 449 (1974).

${ }^{9}$ E. J. Hinch, J. Fluid Mech. 83, 695 (1977).

${ }^{10}$ K. F. Freed and M. Muthukumar, J. Chem. Phys. 68, 2088 (1978).

${ }^{11}$ M. Muthukumar and K. F. Freed, J. Chem. Phys. 70, 5875 (1979).
${ }^{12} \mathrm{~J}$. Rubenstein, J. Fluid Mech. 170, 379 (1986).

${ }^{13}$ S. Kim and W. B. Russel, J. Fluid Mech. 154, 269 (1985).

${ }^{14}$ L. Durlofsky, J. F. Brady, and G. Bossis, J. Fluid Mech. 180, 21 (1987).

${ }^{15}$ J. F. Brady, R. Phillips, J. Lester, and G. Bossis, submitted to J. Fluid Mech.

${ }^{16}$ C. W. J. Beenakker, J. Chem. Phys. 85, 1581 (1986).

${ }^{17}$ R. W. O'Brien, J. Fluid Mech. 91, 17 (1979).

${ }^{18}$ S. Kim and W. B. Russel, J. Fluid Mech. 154, 253 (1985).

${ }^{19} \mathrm{~J}$. F. Brady and L. Durlofsky, submitted to Phys. Fluids.

${ }^{20}$ J. Rubenstein, J. Stat. Phys. 44, 849 (1986). 